Not to appear in Nonlearned J., 45

\title{
Kinematics of gas and stars in circumnuclear star-forming regions of early type spirals
}

\author{
Guillermo F. Hägele, Ángeles I. Díaz and Mónica V. Cardaci ${ }^{1}$ \\ Dpto de Física Teórica, C-XI, Universidad Autónoma de Madrid, 28049 Madrid, Spain \\ and \\ Elena Terlevich ${ }^{2}$ and Roberto Terlevich ${ }^{2}$ \\ INAOE, Tonantzintla, Apdo. Postal 51, 72000 Puebla, México
}

\begin{abstract}
We present high resolution (R 20000) spectra in the blue and the far red of cicumnuclear star-forming regions (CNSFRs) in three early type spirals (NGC 3351, NGC 2903 and NGC 3310) which have allowed the study of the kinematics of stars and ionized gas in these structures and, for the first time, the derivation of their dynamical masses for the first two. In some cases these regions, about 100 to $150 \mathrm{pc}$ in size, are seen to be composed of several individual star clusters with sizes between 1.5 and $4.9 \mathrm{pc}$ estimated from Hubble Space Telescope (HST) images. The stellar dispersions have been obtained from the Calcium triplet (CaT) lines at $\lambda \lambda 8494,8542,8662 \AA$, while the gas velocity dispersions have been measured by Gaussian fits to the $\mathrm{H} \beta$ and [OIII] $\lambda \lambda 5007$ Ålines on the high dispersion spectra. Values of the stellar velocity dispersions are between 30 and $68 \mathrm{~km} / \mathrm{s}$. We apply the virial theorem to estimate dynamical masses of the clusters, assuming that systems are gravitationally bounded and spherically symmetric, and using previously measured sizes. The measured values of the stellar velocity dispersions yield dynamical masses of the order of $10^{7}$ to $10^{8}$ solar masses for the whole CNSFRs. Stellar and gas velocity dispersions are found to differ by about 20 to $30 \mathrm{~km} / \mathrm{s}$ with the $\mathrm{H} \beta$ emission lines being narrower than both the stellar
\end{abstract}

\footnotetext{
${ }^{1}$ XMM-Newton Science Operations Center, ESAC, ESA, POB 78, E-28691 Villanueva de la Cañada, Madrid, Spain.

${ }^{2}$ Research Affiliate at IoA.
} 
lines and the $[\mathrm{OIII}] \lambda \lambda 5007$ Ålines. The twice ionized oxygen, on the other hand, shows velocity dispersions comparable to those shown by stars, in some cases, even larger. We have found indications of the presence of two different kinematical components in the ionized gas of the regions. We have mapped the velocity field in the central kiloparsec of the spiral galaxies NGC 3351 and NGC 2903. For the first object the radial velocity curve shows deviations from circular motions for the ionized hydrogen consistent with its infall towards the central regions of the galaxy at a velocity of about $25 \mathrm{~km} / \mathrm{s}$. For NGC 3310 we present preliminary results of the velocity dispersions for one of the two observed slit position angles, two CNSFRs and the nucleus.

Subject headings: HII regions - galaxies: individual: NGC 2903, NGC 3310, NGC 3351 - galaxies: kinematics and dynamics - galaxies: starburst - galaxies: star clusters.

\section{Observations and data reduction}

The high-resolution blue and far red spectra were acquired as part of an observing run of three nights in 2000. They were simultaneously obtained with ISIS, using the H2400B and R1200R gratings, respectively, on the $4.2 \mathrm{~m}$ William Herschell Telescope. In the blue arm the spectral range is $4780-5200 \AA$ and in the red arm it is $8360-8760 \AA$. The width of the slit is 1 " providing a resolution of $0.21 \AA /$ pix and $0.39 \AA /$ pix, respectively. The spatial resolutions are 0.4 and $0.39 \mathrm{arcsec} / \mathrm{pix}$ for the blue and red arms, respectively.

Three different slit position angles were chosen for NGC 3351 to observe 5 CNSFRs and the nucleus (see Hägele et al. 2007). In the case of NGC 2903 two different slit position angles allow us to study 4 CNSFRs and the nucleus (see Hägele et al. 2008). For NGC 3310 we have observed in two slit positions but we only present preliminary results for one of them. The images were processed and analysed using IRAF routines in the usual manner.

In addition to the galaxy images, observations of 11 velocity template stars were made to provide good stellar reference frames. They are late type giant and supergiant stars which have strong CaT features (see Díaz et al. 1989).

The different methods used to derivate the quantities presented here are explained in detail in Hägele et al. (2007). 


\section{Summary and conclusions}

Stellar velocity dispersions are between 30 and $68 \mathrm{~km} / \mathrm{s}$, about $25 \mathrm{~km} / \mathrm{s}$ larger than those measured for the gas. However, the best Gaussian fits involved two different components for the gas: a "broad component" with a velocity dispersion similar to that measured for the stars, and a "narrow component" with a dispersion lower than the stellar one by about $30 \mathrm{~km} / \mathrm{s}$. This last component seems to have a relatively constant value for all the CNSFRs in each galaxy, with estimated values close to $25 \mathrm{~km} / \mathrm{s}$ for the two gas emission lines. The velocities of the two components of the fits in the CNSFRs of NGC 3351 are the same within the observational errors, but in the cases of NGC 2903 and NGC 3310 we find a shift between the narrow and the broad component that vary between -10 and $35 \mathrm{~km} / \mathrm{s}$ in radial velocity.

The dynamical masses estimated from the stellar velocity dispersion using the virial theorem for the CNSFRs of NGC 3351 are in the range between $4.9 \times 10^{6}$ and $4.8 \times 10^{7} \mathrm{M}_{\odot}$, and is $3.5 \times 10^{7}$ for its nuclear region inside the inner $11.3 \mathrm{pc}$ (Hägele et al. 2007). In the case of NGC 2903 the masses are in the range between $6.4 \times 10^{7}$ and $2.1 \times 10^{8} \mathrm{M}_{\odot}$ for the CNSFRs and is $2.2 \times 10^{7}$ for its nuclear region inside the inner $3.8 \mathrm{pc}$ (Hägele et al. 2008). Masses derived from the $\mathrm{H} \beta$ velocity dispersions under the assumption of a single component for the gas would have been underestimated by factors between approximately 2 to 4 .

Masses of the ionising stellar clusters of the CNSFRs have been derived from their $\mathrm{H} \alpha \mathrm{lu}-$ minosities (Planesas et al. 1997) under the assumption that the regions are ionisation bound and without taking into account any photon absorption by dust. Their values for NGC 3351 are between $8.0 \times 10^{5}$ and $2.5 \times 10^{6} \mathrm{M}_{\odot}$ for the starforming regions, and is $6.0 \times 10^{5}$ for the nucleus (Hägele et al. 2007). And for the regions of NGC 2903 they are between $3.3 \times 10^{6}$ and $4.9 \times 10^{6} \mathrm{M}_{\odot}$, and is $2.1 \times 10^{5}$ for its nucleus (Hägele et al. 2008). These values are comparable to that derived by Gonzalez-Delgado et al. (1995) for the circumnuclear region A in NGC 7714. Therefore, the ratio of the ionising stellar population to the total dynamical mass is between 0.01 and 0.16 .

Derived masses for the ionised gas vary between $7.0 \times 10^{3}$ and $8.7 \times 10^{4} \mathrm{M}_{\odot}$ for the CNSFRs of NGC 3351 (Hägele et al. 2007), and is $2 \times 10^{3} \mathrm{M}_{\odot}$ for the nucleus, and between $6.1 \times 10^{4}$ and $1.3 \times 10^{5} \mathrm{M}_{\odot}$ for the regions and is $2 \times 10^{3} \mathrm{M}_{\odot}$ for the nucleus of NGC 2903 (Hägele et al. 2008). These values are also comparable to that derived by Gonzalez-Delgado et al. (1995).

For NGC 3351, the rotation velocities derived for both stars and gas are in reasonable agreement, although in some cases the gas shows a velocity slightly different from that

of the stars (Hägele et al. 2007). The rotation curve corresponding to the position going through the centre of the galaxy shows maximum and minimum values at the position of 
the circumnuclear ring, as observed in other galaxies with CNSFRs (Díaz et al. 1999, and reference therein). The differences in velocity between gas and stars can be interpreted as motions of the ionised hydrogen deviating from rotation and consistent with a radial infall to the central regions of the galaxy. Our results are consistent with those found by Rubin et al. (1975) and would yield an infall velocity of about $25 \mathrm{~km} / \mathrm{s}$.

On the other hand, the observed stellar and [OIII] velocities of NGC 2903 are in good agreement, while the $\mathrm{H} \beta$ measurements show shifts similar to those find between the narrow and the broad components (Hägele et al. 2008). This different behavior can be due to that the position of the single Gaussian fits are dominated by the broad component in the case of the [OIII] emission line while in the case of the $\mathrm{H} \beta$ are dominated by the narrow one. Again, the rotation curve corresponding to the position going through the nucleus shows maximum and minimum values at the positions of the circumnuclear regions.

\section{REFERENCES}

Díaz, A. I., Terlevich, E., \& Terlevich, R. 1989, MNRAS, 239, 325

Díaz, R., Carranza, G., Dottori, H., \& Goldes, G. 1999, ApJ, 512, 623

González-Delgado, R. M., Pérez, E., Díaz, A. I., García-Vargas, M. L., Terlevich, E., \& Vílchez, J. M. 1995, ApJ, 439, 604

Hägele, G. F., Díaz, Á. I., Cardaci, M. V., Terlevich, E., \& Terlevich, R. 2007, MNRAS, 378,163

Hägele, G. F., Díaz, Á. I., Cardaci, M. V., Terlevich, E., \& Terlevich, R. 2008, MNRAS, in preparation

Planesas, P., Colina, L., \& Pérez-Olea, D. 1997, A\&A, 325, 81

Rubin, V. C., Peterson, C. J., \& Ford, W. K., Jr. 1975, ApJ, 199, 39

This preprint was prepared with the AAS LATEX macros v5.2. 